\title{
Reference Point Detection for Arch Type Fingerprints
}

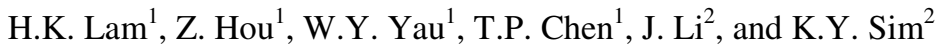 \\ ${ }^{1}$ Computer Vision and Image Understanding Department \\ Institute for Infocomm Research, A*STAR \\ (Agency for Science, Technology and Research), \\ Fusionopolis, Singapore \\ \{hklam, zhou\}@i2r.a-star.edu.sg \\ ${ }^{2}$ School of Electrical and Electronic Engineering \\ Nanyang Technological University \\ Singapore
}

\begin{abstract}
Reference point detection is an important task in the design of an automated fingerprint identification system. Many algorithms have emerged with acceptable results but are mostly suitable for non-arch type fingerprint. It still remains as a challenging problem to reliably identify reference points for fingerprints of the arch type. A topological method is presented in this paper to detect reference points in arch type fingerprint images. To evaluate the performance, 400 arch type fingerprint image pairs in the NIST DB4 database are utilized. The alignment accuracy on average is about 35 pixels in distance and 9 degrees in orientation, which is very well comparable with respect to state-ofthe-arts as designed for non-arch type fingerprints.
\end{abstract}

\section{Introduction}

In the design of an automated fingerprint identification system (AFIS), a frequently encountered problem is to reliably locate a reference point. An accurately located reference point could substantially improve the efficiency of an AFIS [1]. Usually the reference point is selected from a list of singular points (core/delta point) [2] located in the acquired fingerprint image. Singular points usually refer to discontinuities in the fingerprint orientation field and are important features for fingerprint images as they can effectively be used for fingerprint classification, registration, alignment and etc.

However, it is not trivial to locate singular points accurately and precisely as the result can be affected by a number of factors such as noise, wetness and dryness of the finger, and the type of fingerprint class (arch or non-arch). Nevertheless, the singular point can be said to be more easily located in a non-arch type fingerprint compared to an arch type fingerprint. This is because in the case of arch type fingerprint, the ridges are almost parallel across the whole fingerprint image and as a result, the conventional concept of singular points is not well defined.

Thus it is not surprising to witness that most efforts for reference point detection focus on fingerprints of non-arch types and ignore the arch types [3-6]. Although compared with other fingerprint types, the percentage of people with arch type 
fingerprint is smaller, in absolute numbers, they are still quite large. For techniques on reference point detection to be practically useful in an AFIS, there is no reason to ignore the arch type fingerprint, in particular when considering the high demand on security in the era that terrorism remains a severe threat on people's daily life and economic prosperity.

The rest of the paper is organized as follows: Section 2 will detail our method of locating reference points in arch type fingerprint images, Section 3 will show our results and compare with some of the well known singular point detection algorithm as designed for non-arch type fingerprint images and Section 4 concludes the paper with a brief discussion on future direction.

\section{Methodology}

The acquired fingerprint image is firstly segmented to extract the foreground where the fingerprint structure is present. Then fingerprint orientation is estimated using the method in [7] to obtain the orientation map. Fig. 1 shows the original image and the orientation image where the dot (in orientation image) indicates background. The region of interest in the following processing will be limited to the foreground where the fingerprint structure is present.

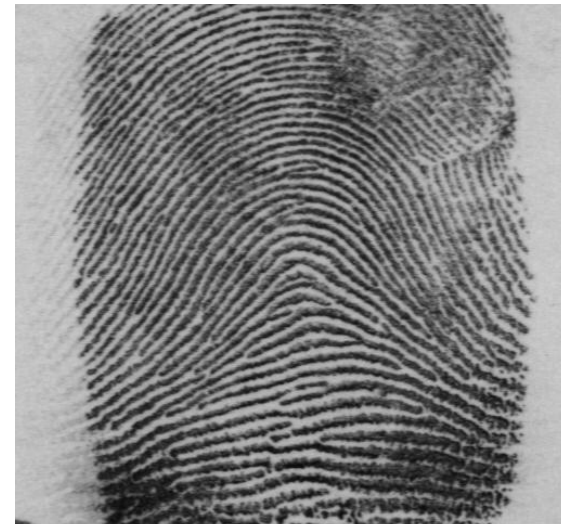

(a)

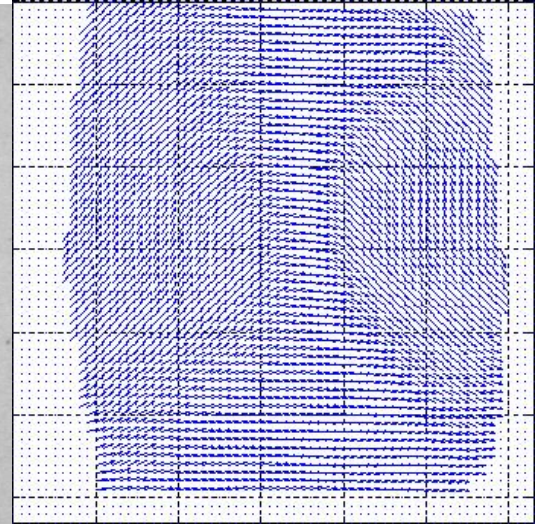

(b)

Fig. 1. (a) An arch type fingerprint image, (b) orientation image

\subsection{Finding Symmetry Line of Fingerprint Structure}

Compared with non-arch type fingerprints, the most distinctive feature of the arch type ones is the line symmetry for the overall fingerprint structure. To utilize this topological feature for characterizing the arch type fingerprint, the orientation of the fingerprint image is further processed to separate it into two levels and to detect the symmetry line from this segmentation map.

Toward that end, a threshold to segment the orientation map of the fingerprint image must be calculated. This is done automatically by first obtaining an orientation 
histogram from the orientation map and then finding a point such that the area of each region of the thresholded orientation histogram is equal. Fig. 2 shows the orientation histogram of the fingerprint image in Fig. 1 and the automatically calculated threshold drawn in red. The threshold is applied to the orientation map to obtain a two level segmentation map. Then morphological processing is applied to derive a smooth segmentation map, as shown in Fig. 3(a) for example.

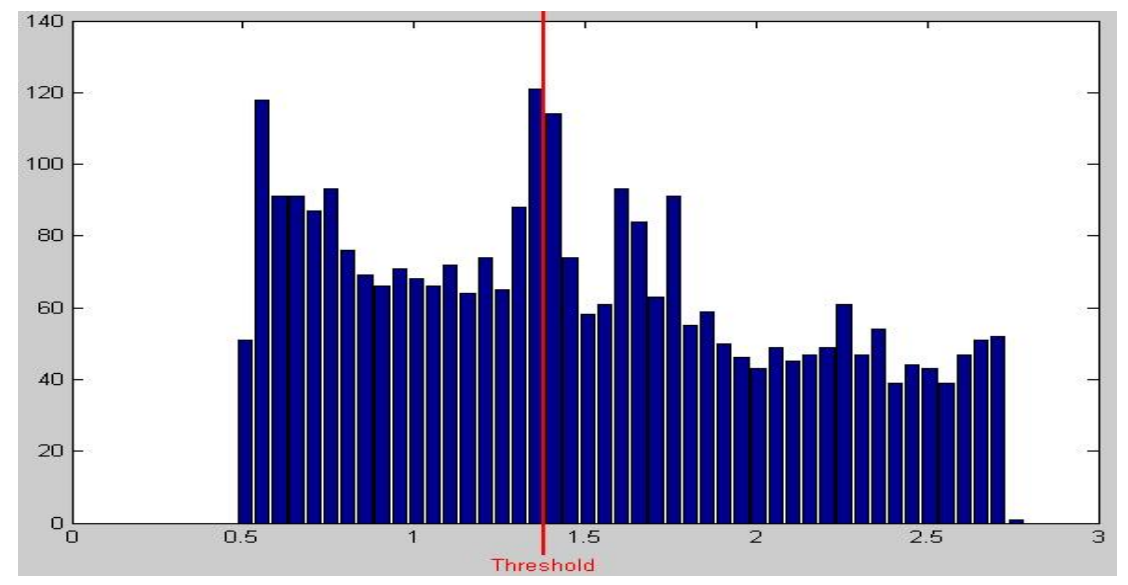

Fig. 2. Two level thresholding on the orientation histogram

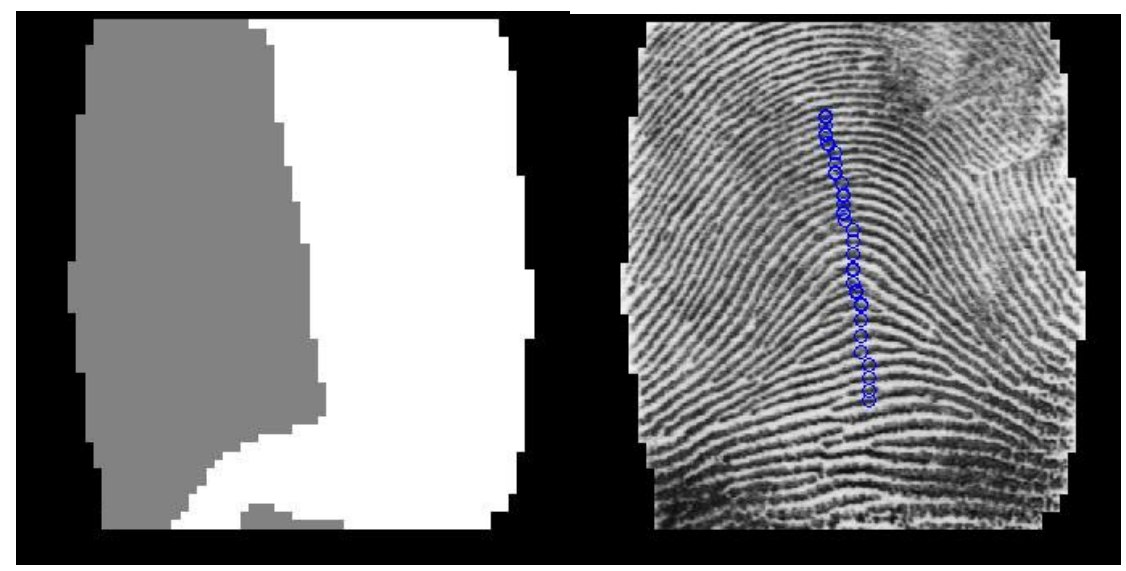

(a)

(b)

Fig. 3. (a) A two level segmentation of the orientation field for an arch type fingerprint image. (b) The major symmetric line as identified from boundaries between the segmented regions in (a) and represented as blue circles.

Very often, the segmented orientation map does not produce two perfectly symmetric regions, as seen from Fig. 3(a) for instance. Through intensive investigation, we observe that frequently there will be one major boundary line that runs through the 
segmentation map. Thus, we can confidently select the longest boundary line as the symmetry line of the fingerprint image.

Firstly, the coordinates of the boundary points are traced according to 8neighbourhood connectivity and labeled as a group. For instance, there are two boundary lines in Fig. 3(a). After all boundary points are labeled, the line that is longest and cuts closest along the centre of the fingerprint structure will be the symmetry line of the fingerprint image. Fig. 3(b) shows the result of the identified symmetric line from fig. 3(a). For convenience, the point set in the line is denoted as $\ell=\left\{P_{i} \mid i=N_{\ell}\right\}$, where $N_{\ell}$ is the number of the points.

\subsection{Locate Reference Point and Orientation}

After identifying the symmetric line, we are ready to define the reference point for arch type fingerprints. As aforementioned, line symmetry is the most significant feature of arch type fingerprints, which distinguishes arch type fingerprints from non-arch type ones in terms of global pattern characterization. For non-arch type fingerprints, singular points represent the most evident global features, contain rich information for fingerprint classification and can reliably be used as candidate of reference point.

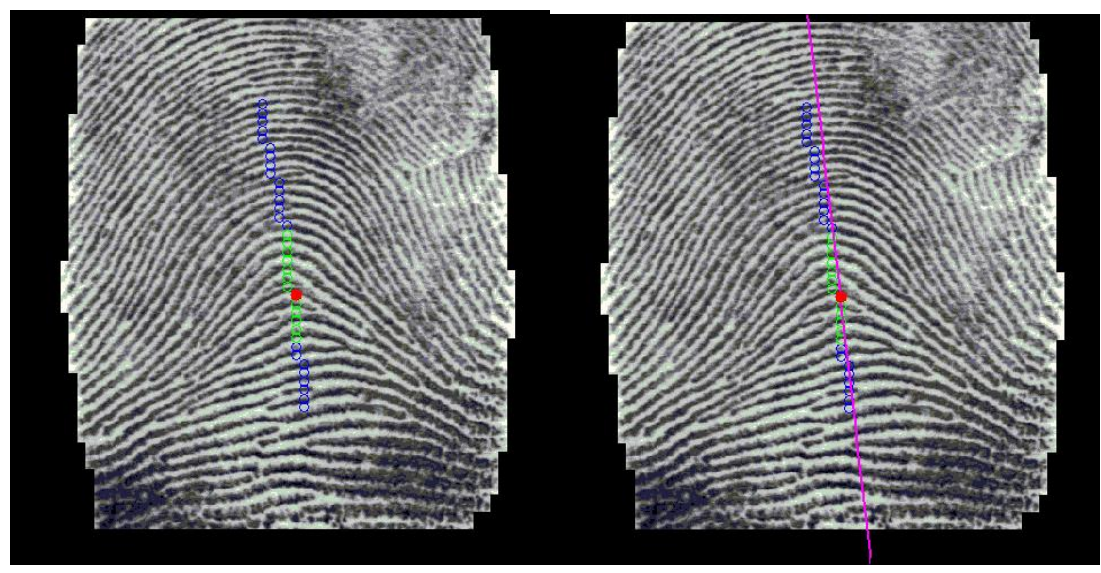

(a)

(b)

Fig. 4. (a) The located reference point (in red) has the greatest change of orientation angle along the symmetric line. (b) The green circles are used to calculate the orientation of the reference point. The magenta line represents the orientation of the reference point.

Although singular points are not well defined for arch type fingerprints, we argue that candidate of reference point detection should be determined from the points that most fundamentally characterize the arch type fingerprints. With this in mind, we define the reference point of an arch type fingerprint as the point in the above identified symmetric line with the greatest change in direction represented using: 


$$
P^{*}=\max _{i \in N_{\ell}}\left\|\nabla \varphi_{i}\right\|
$$

where $\varphi_{i}$ is the orientation at the i-th pixel. Fig. 4(a) shows the detected reference point for an arch type fingerprint.

Besides finding the location of the reference point, the orientation of the reference point is also estimated from the identified symmetric line. Towards that end, the points within a distance from the located reference point $P^{*}$ are selected, as denoted by $P_{k}, k=1, \cdots, n$. Then a straight line $y=\beta_{0}+\beta_{1} x$ is fitted among the points and the parameters are estimated using the least square error method. The orientation of the reference point is estimated as

$$
\varphi^{*}=\arctan \beta_{1}
$$

Fig. 4(b) shows the result of obtaining the orientation of the reference point from the symmetric line.

\section{Results}

\subsection{Evaluation Criteria}

To evaluate the performance of a method for fingerprint reference point detection, one usually compares the detected results with respect to the ground truth which is derived from experts' labeling. However, this kind of strategy is problematic for the case of arch-type fingerprints. As aforementioned, the concept of singular point is not well defined for arch-type fingerprints, thus there is a large degree of uncertainty for fingerprint experts to manually identify a point as the reference point. To tackle this issue, we propose here to validate the performance through matching as detailed in the following:

1. Two fingerprint images from the same subject are matched and the correspondence of matched minutia pairs is established;

2. The same two fingerprint images are aligned using the reference points;

3. The average matching minutiae distance difference and angle difference of the normalized set is calculated and used as performance index.

In order to have a fair comparison of our algorithm and due to the lack of good reference point locating algorithm for arch type fingerprint images in the literature, we compare our result for arch type fingerprint images to that of well know algorithm for non-arch type fingerprint images. For non-arch type fingerprint images, the reference point was located using the complex filter [8]. For arch type fingerprint images, our method was used to locate the reference point.

The database used for testing is NIST DB4 where there are 2000 pairs of images. $20 \%$ of the images are arch-type images. Out of the rest of the 1600 pairs of images, only 1280 pairs of the non-arch type fingerprint images are usable because the complex filter is unable to find a reference point in one of either image. The 1680 pairs of fingerprint images are matched using software [9] to obtain the matching minutiae for use in computing the performance of the reference point locating algorithms. 
Table 1. Results of our reference point detection algorithm for arch type fingerprint images and comparison with respect to the complex filter results on non-arch type fingerprint images

\begin{tabular}{|l|c|c|}
\hline & $\begin{array}{l}\text { Complex filter method } \\
\text { on non-arch type image }\end{array}$ & $\begin{array}{l}\text { Our proposed method } \\
\text { on arch type image }\end{array}$ \\
\hline $\begin{array}{l}\text { Average matching minutiae } \\
\text { normalized distance difference }\end{array}$ & 28.58 pixels & 35.18 pixels \\
\hline $\begin{array}{l}\text { Average matching minutiae } \\
\text { normalized angle difference }\end{array}$ & 9.41 degrees & 8.79 degrees \\
\hline
\end{tabular}

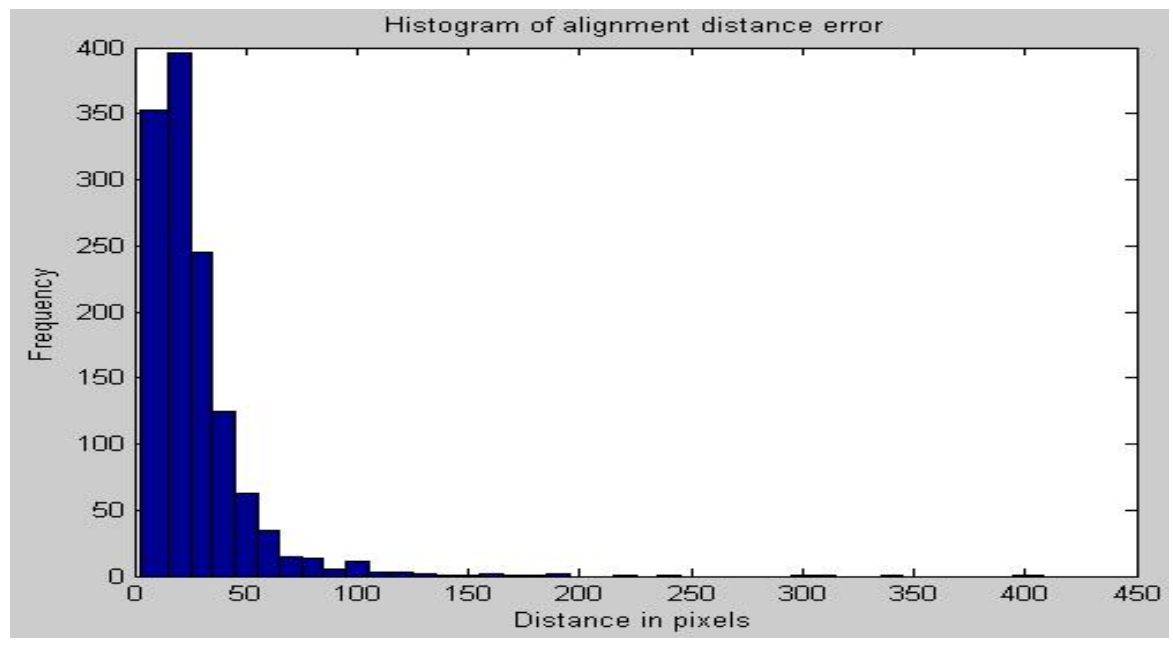

(a)

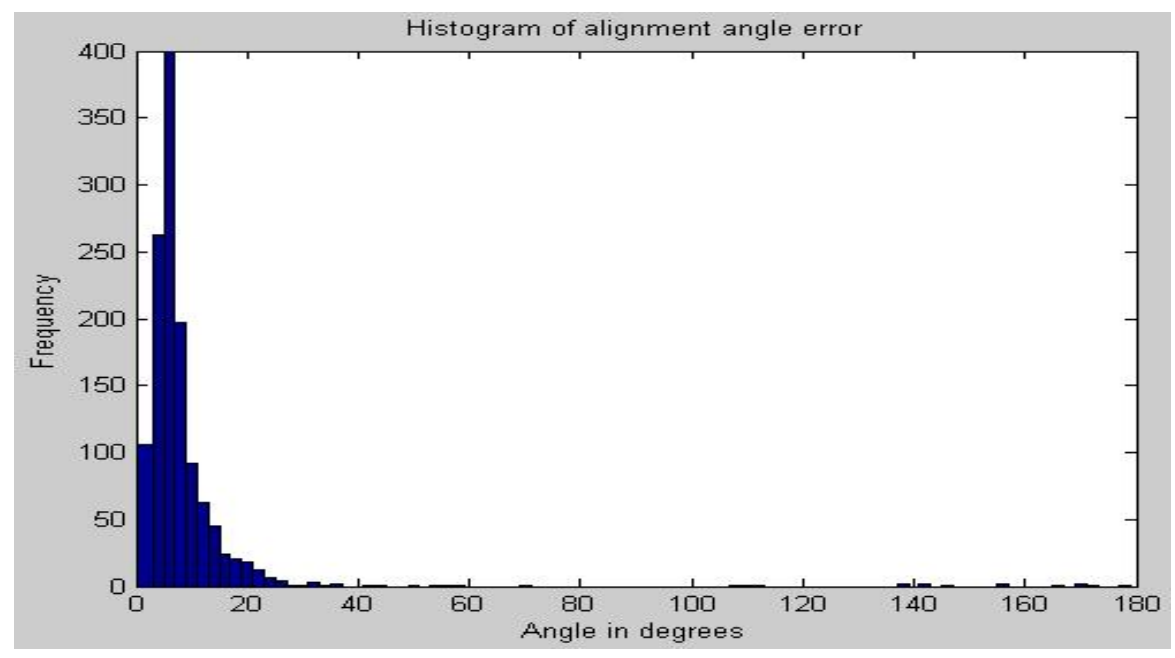

(b)

Fig. 5. (a) Histogram showing the distribution of distance difference. (b) Histogram showing the distribution of angle difference. Algorithm used is complex filter for non-arch type images only. 


\subsection{Experimental Results}

The result is summarized in Table 1 ( $3^{\text {rd }}$ column). The alignment accuracy of the proposed method is about 35 pixels in distance and 9 degrees in orientation. Table 1 also shows the result of the complex filter method $\left(2^{\text {nd }}\right.$ column $)$. From the table, it is clear that our algorithm is able to accurately determine the position and orientation of the

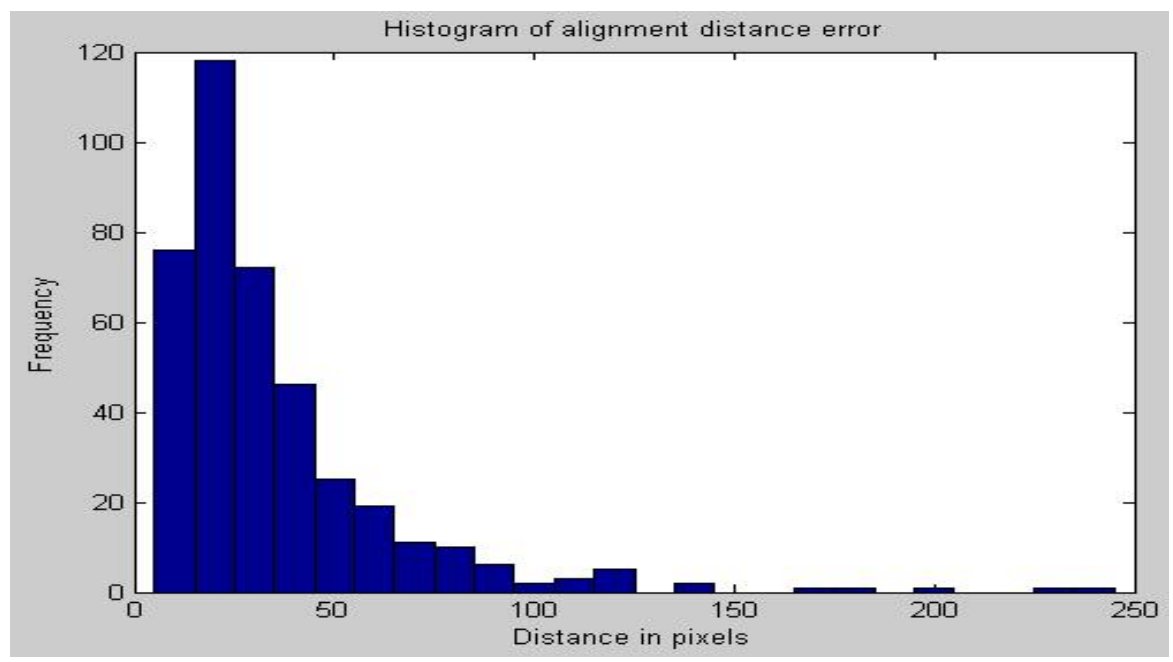

(a)

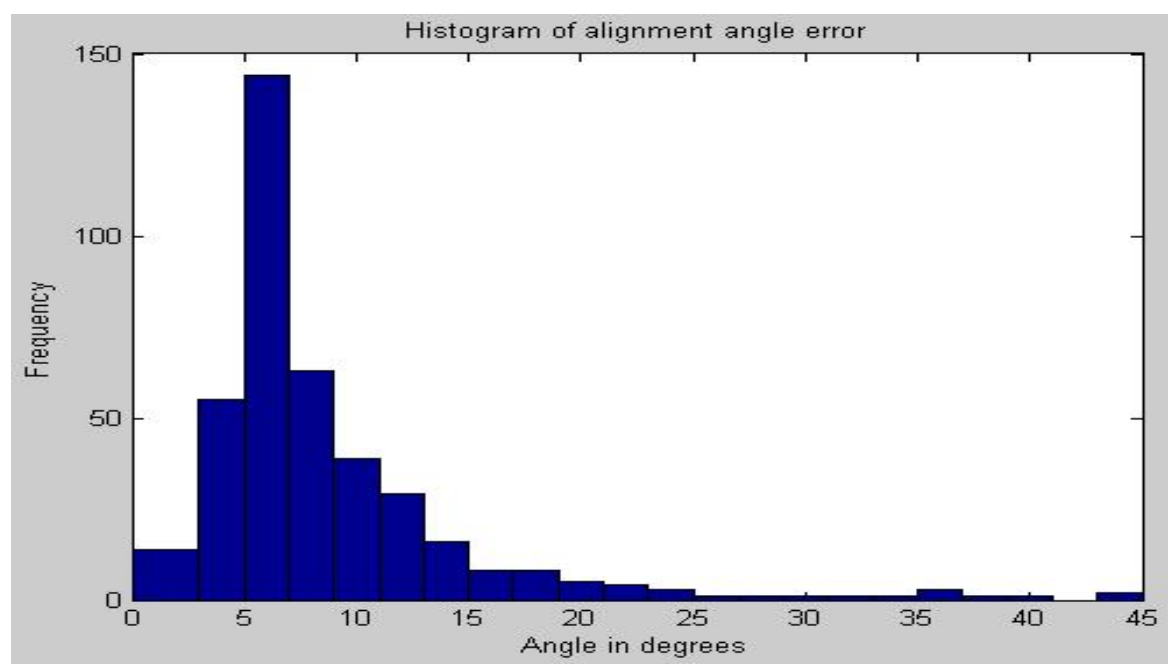

(b)

Fig. 6. (a) Histogram showing the distribution of distance difference. (b) Histogram showing the distribution of angle difference. Algorithm used is our proposed method for arch type images only. 

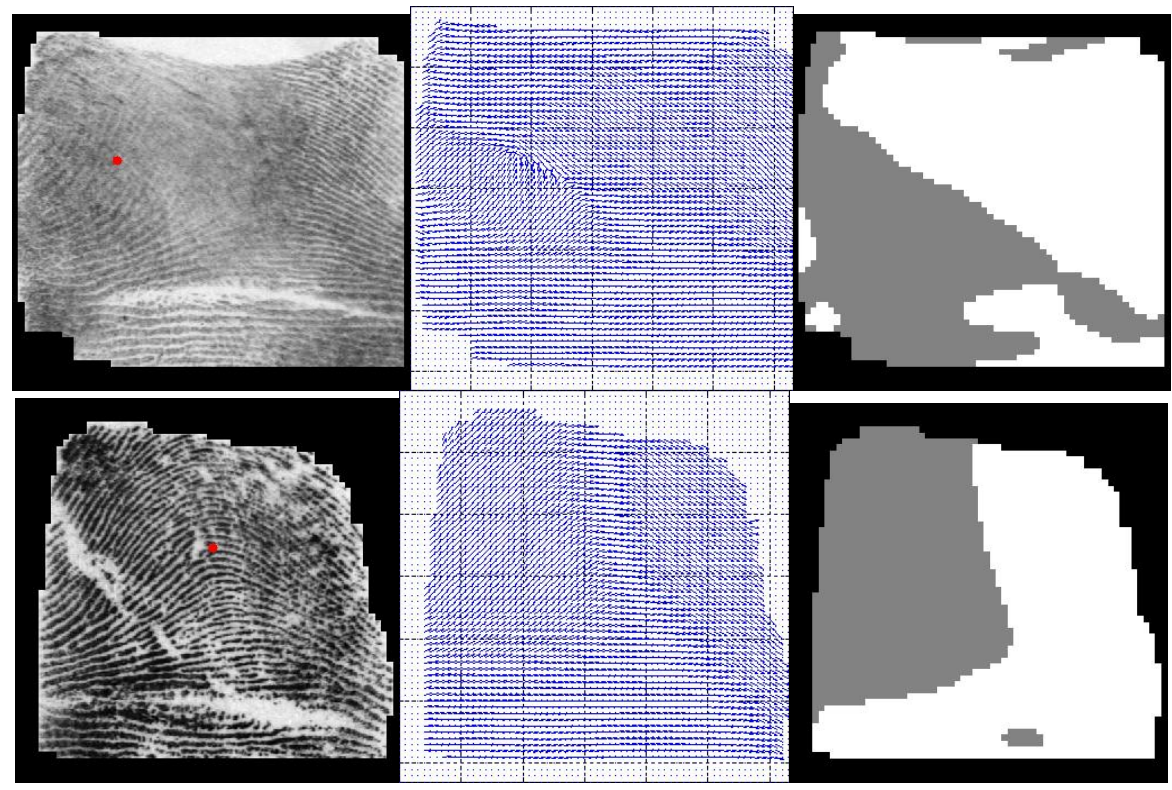

Fig. 7. Two instance of the same finger. The series on the top shows noise in the image causing the orientation map to be computed incorrectly and subsequently segmented wrongly. The series on the bottom shows a proper segmented orientation map and thus correctly detected reference point. Alignment error is large in this case.

reference points in arch type fingerprint images. The result is at least comparable to well known algorithm for locating reference points in non-arch type fingerprint images. The result of the proposed method is also comparable with respect to methods in $[10,11]$ (as evaluated using the FVC2000 DB2 database) when considering that the image quality of FVC2000 DB2 is much better than the NIST DB4 used here and either [10] or [11] rejects about 10 images in their result.

For a more detailed comparison, the distributions of the error in both distance and angle are plotted in Fig. 5 (for the complex filter method) and Fig. 6 (for the proposed method) respectively. From Fig. 5, one can see that the alignment error is fairly small for most images and the occurrence of large error is mainly due to the poor image quality, thus it is reasonable to align non-arch type fingerprints using the complex filter method. Similar observation can be found in Fig. 6, where proposed method is satisfactory for most arch type fingerprints with reasonable quality. From Figs. 5 and 6, it can be expected that the performance of fingerprint alignment can substantially be improved by fingerprint enhancement, which is out of the scope of this presentation.

For the proposed method, a reasonable image quality means that the segmentation of the orientation pattern can be reliably derived. Otherwise, large alignment error will happen. Fig. 7 shows a typical example on how the image quality affects the estimation of the orientation field as well as the result of segmentation.

Both methods are implemented in Matlab. The complex filter takes around 2.0s to complete the reference point detection, while our algorithm takes about $0.05 \mathrm{~s}$. 


\section{Conclusion}

To summarize, we have presented a method to locate reference points in arch type fingerprint images. The method starts from the detection of the symmetric line, which is topologically the most remarkable feature in arch type fingerprints, and defines the reference point as the point in the symmetric line with largest change in orientation. The orientation of the reference point is also estimated from the identified symmetric line. The method has been evaluated using the 400 arch type fingerprint image pairs in the NIST DB4 database. Our alignment accuracy is about 35 pixels in distance and 9 degrees in orientation, which is well comparable to the complex filter method as targeted for nonarch type fingerprint images. The experiment demonstrates that the proposed method is simple but is it fast and accurate. With this method, it can be expected that the technique of reference point detection can be integrated into practical AFIS to improve efficiency.

It should be pointed out that the results for our algorithm are based on a coarse level analysis ( $8 \times 8$ blocks). Thus, further processing can be done to locate the reference points to pixel accuracy. In addition, some images are too wet or very noisy, and significantly degrade the performance of the proposed method. Therefore more reliable methods for fingerprint enhancement and orientation calculation may be researched on to improve the location accuracy.

\section{References}

1. Chikkerur, S., Ratha, N.: Impact of singular point detection on fingerprint matching performance. In: 4th IEEE workshop on Automatic Identification Advanced Technologies, pp. 207-212 (2005)

2. Jain, A.K., Prabhakar, S., Hong, L., Pankanti, S.: Filterbank-based fingerprint matching. IEEE Trans. Image Processing 9, 846-859 (2000)

3. Kitiyanan, N., Havlicek, J.P.: Modulation domain reference point detection for fingerprint recognition. In: 6th IEEE Southwest Symp. on Image Analysis and Interpretation, pp. 147151 (2004)

4. Zhang, H., Yin, Y., Ren, G.: An improved method for singularity detection of fingerprint images. In: Li, S.Z., Lai, J.-H., Tan, T., Feng, G.-C., Wang, Y. (eds.) SINOBIOMETRICS 2004. LNCS, vol. 3338, pp. 516-524. Springer, Heidelberg (2004)

5. Ohtsuka, T., Kondo, A.: A new core and delta detection for fingerprints using the extended relation graph. In: IEICE Trans. on Fundamentals of Electronics, Communications and Computer Sciences, pp. 2587-2592 (2005)

6. Huang, C., Liu, L., Douglas Hung, D.C.: Fingerprint analysis and singular point detection. Pattern Recognition Letters 15, 1937-1945 (2007)

7. Wang, Y., Hu, J., Phillips, D.: A Fingerprint Orientation Model Based on 2D Fourier Expansion (FOME) and Its Application to Singular-Point Detection and Fingerprint Indexing. Trans. on Pattern Analysis and Machine Intelligence 29(4), 573-585 (2007)

8. Nilsson, K., Bigun, J.: Complex filters applied to fingerprint images detecting prominent symmetry points used for alignment. In: Tistarelli, M., Bigun, J., Jain, A.K. (eds.) ECCV 2002. LNCS, vol. 2359, pp. 39-47. Springer, Heidelberg (2002)

9. http://www.fingerprint.nist.gov/NBIS/index.html

10. Jiang, X., Liu, M., Kot, A.C.: Reference point detection for fingerprint recognition. In: Intl. Conf. in Pattern Recognition, pp. 540-543 (2004)

11. Liu, T., Zhang, C., Hao, P.: Fingerprint reference point detection based on local axial symmetry. In: Intl. Conf. in Pattern Recognition, pp. 1050-1053 (2006) 